NASA Technical Memorandum 102121

\title{
On the Dynamic Response of Pressure Transmission Lines in the Research of Helium-Charged Free Piston Stirling Engines
}

Eric L. Miller

Sverdrup Technology Inc.

NASA Lewis Research Center Group

Cleveland, Ohio

and

James E. Dudenhoefer

National Aeronautics and Space Administration

Lewis Research Center

Cleveland, Ohio

Prepared for the

24th Intersociety Energy Conversion Engineering Conference

cosponsored by the IEEE, AIAA, ANS, ASME, SAE, ACS, and AIChE Washington, D.C., August 6-11, 1989 
ON THE DYNAMIC RESPONSE OF PRESSURE TRANSMISSION LINES

IN THE RESEARCH OF HELIUM-CHARGED FREE PISTON STIRLING ENGINES

Eric L. Miller

Sverdrup Technology, Inc.

NASA Lewis Research Center Group

Cleveland, Ohio 44135

and

James E. Dudenhoefer

National Aeronautics and Space Administration

Lewis Research Center

Cleveland, Ohio 44135

ABSTRACT

In free piston Stirling engine research the integrity of both amplitude and phase of the dynamic pressure measurements is critical to the characterization of cycle dynamics and thermodynamics. It is therefore necessary to appreciate all possible sources of signal distortion when designing pressure measurement systems for this type of research. This paper discusses the signal distortion inherent to pressure transmission lines. Based on results from classical analysis, guidelines are formulated to describe the dynamic response properties of a volume-terminated transmission tube for applications involving helium-charged free piston Stirling engines. The scope and limitations of the dynamic response analysis are considered.

\section{INTRODUCTION}

It is often impossible to flush-mount a dynamic pressure transducer in the expansion space or the compression space of a free-piston Stirling engine. In some cases, direct exposure to the severe gas temperatures would threaten to destroy the transducer: In other cases, the engine design precludes straightforward transducer access. When flush-mounting is not possible, the transducer is physically removed from the working space via a volume-terminated transmission tube (some termination volume at the instrument end of the tube is an inevitable consequence of transducer installation.)

In these situations, the instrumentation engineer must ensure that errors introduced in the pressure signal by the transmission tube remain within acceptable tolerances. In Stirling engine research, the error tolerances on dynamic pressure measurements are particularly stringent since small errors in either amplitude or phase of the measurement can cause significant errors in important calculations, such as the $\mathrm{PV}$ power.

This paper will develop some useful guidelines for the design of pressure transmission lines for use in helium-charged free piston engine applications.
DEFINITION OF SYMBOLS

The following symbols are defined in order to facilitate the discussion of pressure transmission lines and their use in Stirling engine research:

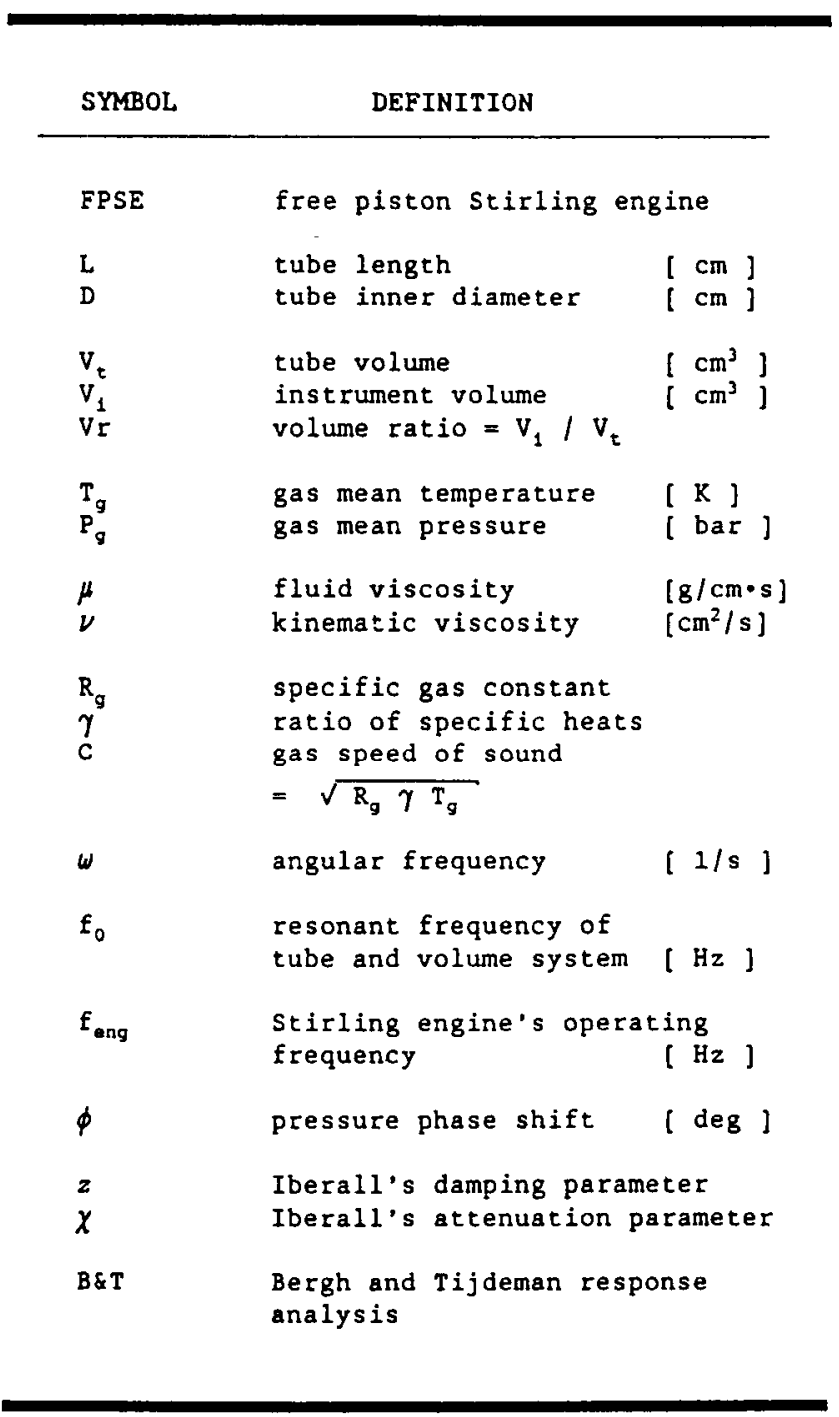




\section{DYNAMIC RESPONSE OF A VOLUME-TERMINATED TUBE}

The general analysis of oscillatory pressure transmission through a volume-terminated tube is a classical problem in Instrumentation engineering. Since a transmission tube will distort both the amplitude and phase of a dynamic pressure measurement, it is necessary to perform a complete dynamic-response analys is to theoretically bound the measurement errors.

To date, the most comprehensive analytical treatment of this problem has been presented by Iberali[1]. In his 1950 treatise, Iberall solved the fundamental equations of fluid flow in order to establish how an oscillatory pressure is transmitted through a tube and volume system. The resulting dynamic response curves depict both the amplitude distortion and the phase shift as a function of oscillation frequency.

Figure 1 represents a typical two-part response curve derived from Iberall's mathematical technique:

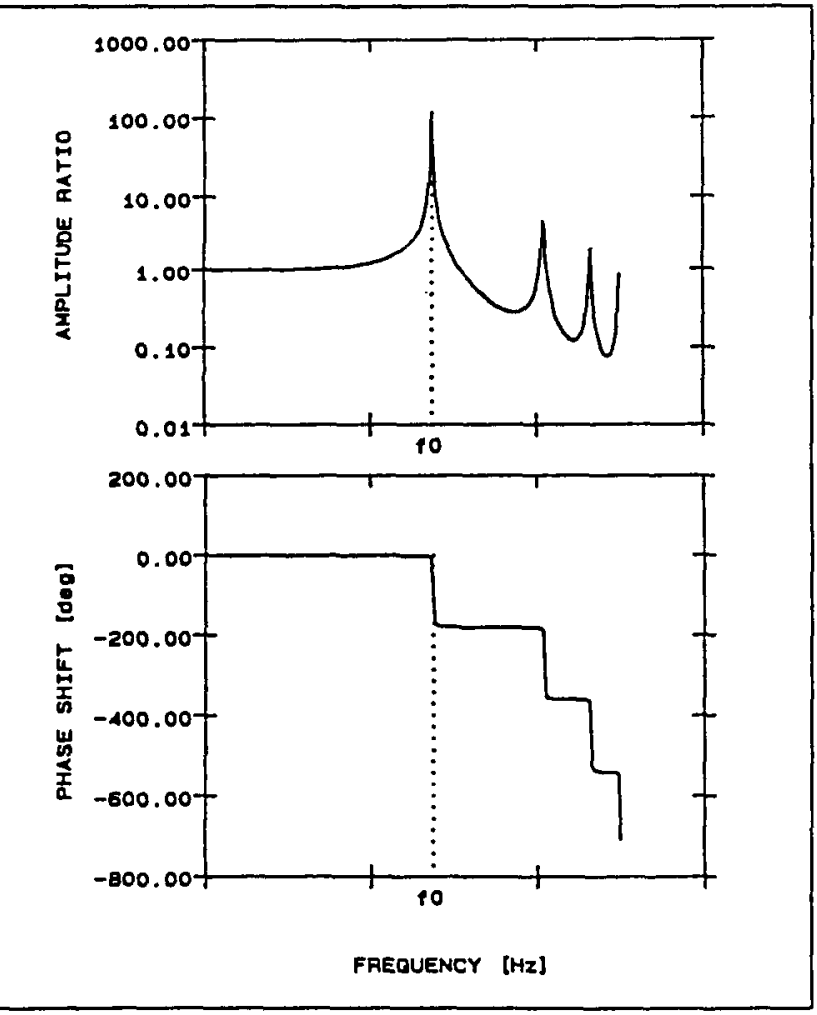

Figure 1: TYPICAL DYNAMIC RESPONSE 8EHAVIOR FOR PRESSURE TRANSMISSION THROUGH A VOLUME-TERMINATED TUBE

It can be seen that for frequencies which are low with respect to the primary resonant frequency, the dynamic response curves are reasonably flat; dynamic pressures at these frequencies will be transmitted predictably with little distortion. It is necessary to take advantage of this flat portion of the response curve when designing a pressure transmission system. The instrumentation engineer needs to be equipped with basic analytical tools in order to perform such design.
There are a number of available computer algorithms based on Iberall's results which have been successfully validated by laboratory experiments. One example is the code presented by Goldschmied[2]. Another is the algorithm developed at the NASA Lewis Research Center by Nyland, Englund and Anderson [3]; the NASA code implements a method presented by Bergh and Tijdeman [4] which extends Iberall's mathematics to handle more complicated tube and volume geometries.

In the Stirling Engine Branch at NASA Lewis, the Bergh and Tijdeman code has become a standard tool in the evaluation of pressure instrumentation systems. This paper will draw upon computer results from the $B \& T$ code along with analytical results from Iberall's paper to demonstrate basic properties of pressure transmission response.

\section{DESCRIPTION OF THE UNDERDAMPED FLOW REGIME}

In general, the response curves for a tube and volume system will be influenced by tube length. tube diameter, volume ratio and gas properties (which are a function of gas mean temperature and gas mean pressure.) However, an evaluation of pressure transmission lines in existing heliumcharged free-piston research engines has yielded some interesting observations: although the resonant frequencies of these lines are strongly affected by tube length, volume ratio and gas mean temperature, they are essentially independent of tube diameter and gas mean pressure.

Using results from the Iberall analysis, it will be shown that this behavior is characteristic of transmission tube response when the working fluid in the tube is 'underdamped'. First, the conditions for underdamped flow will be discussed; and it will be shown that the helium in pressure transmission lines of typical FPSE applications will behave as an underdamped fluid.

The dimensionless parameter used by Iberal1[1] to indicate the amount of damping in a fluid flow is $z$ :

$$
z=\frac{D^{2} \omega}{4 \nu}=\frac{D^{2} \pi f}{2 \nu} \quad \text { [ eq. } 1 \text { ] }
$$

Here, $\nu$ is the kinematic gas viscosity, and $f$ is the frequency of the pressure oscillation. The fluid is considered underdamped when $z \geq 100$.

For a given FPSE application, $f$. is fixed by the engine's operating frequency, and $\nu$ is a fixed function of gas mean temperature and gas mean pressure; it then follows that the tube diameter, $D$. is the only independent variable in this equation. If $z$ is replaced with the threshold value of 100 , equation 1 can be rearranged to give an expression for the minimum tube I.D. which will guarantee underdamped gas flow in the tube:

$$
D_{\text {min }}=\sqrt{\frac{200 \nu_{\text {gon }}}{\pi f_{\text {eng }}}} \quad \text { [ eq. 2] }
$$


Table I calculates $D_{\text {min }}$ for a helium-charged engine with $f_{\text {ong }}=100 \mathrm{~Hz}$ at various values of gas mean temperature and pressure.

\begin{tabular}{rlll}
{$\left[\begin{array}{l}\mathrm{T}_{g} \\
{[\mathrm{~K}]}\end{array}\left[\begin{array}{l}\mathrm{P}_{\mathrm{g}} \\
\text { bar }]\end{array}\right.\right.$} & $\begin{array}{c}\nu \\
\left.\mathrm{cm}^{2} / \mathrm{s}\right]\end{array}$ & $\begin{array}{c}\mathrm{D}_{\text {min }} \\
{[\mathrm{cm}]}\end{array}$ \\
\hline 300 & 150 & .0088 & .075 \\
750 & 150 & .0390 & .157 \\
1050 & 150 & .0676 & .207 \\
300 & 100 & .0129 & .091 \\
750 & 100 & .0585 & .193 \\
300 & 50 & .0628 & .200
\end{tabular}

TABLE I: $D_{\min }$ looHz vs. $T_{g}$ and $P_{g}$ for Helium
$(\nu$ values from gas tables $)$

It can be seen that for many conventional tube sizes $\left(.32 \mathrm{~cm}, .64 \mathrm{~cm}\right.$ etc.), $\mathrm{D}_{\text {tube }}$ will exceed $\mathrm{D}_{\mathrm{min}}$ in $100 \mathrm{~Hz}$ FPSE applications; thus the helium is underdamped.

This paper will argue that underdamped flow is not only achievable in most FPSE applications, it is also desirable. The analysis of transmission tubes becomes simplified for low damping; and more important, the pressure phase shifts are considerably less severe when the gas flow is underdamped.

In consequence, the instrumentation engineer should select tubes for a pressure transmission line which ensure underdamped helium flow at the specific application frequency. Equation 2 provides the necessary guidance.

\section{THE PRIMARY RESONANCE FREQUENCY}

As previously illustrated in figure 1 , the most important design consideration for a pressure transmission system is the primary resonant frequency. For a complete description of resonance phenomena in a volume-terminated transmission tube, the reader is referred to Iberall's complete dissertation[1]; The basic results developed for the underdamped flow regime, however, represent a significant simplification of the generalized response analysis. Two of these simplified results can be sumnarized as follows:

1) The primary resonant frequency for a transmission tube at a fixed volume ratio will vary in proportion to the quantity $\mathrm{C} / \mathrm{L}$. Here, $C$ represents the gas speed of sound and $L$ is the tube length.

2) For a fixed value of $\mathrm{C} / \mathrm{L}$, the frequency of primary resonance is solely a function of the volume ratio. The theory does not provide a simple analytical expression for this function; for convenience, it will be denoted by $\mathbf{G}\left(V_{r}\right)$ in this paper.

A combination of these results implies that the primary resonant frequency can be described by the following proportionality:

$$
f_{0} \propto \frac{C}{L} G(V r) \quad[\text { eq. } 3]
$$

Or, since the gas speed of sound, $C$, is proportional to the square root of the gas mean temperature:

$$
f_{0} \propto \frac{\sqrt{T_{g}}}{L} G(\mathrm{Vr}) \quad[\text { eq. } 4]
$$

This represents a generalized expression for the primary response resonance when the fluid flow in the transmission tube is underdamped. In this formula there are no explicit dependencies on tube diameter or gas mean pressure; a result which is consistent with the observations mentioned earlier for transmission lines used in helium-charged FPSE'S.

A specific equation for underdamped helium systems can be developed in the form of equation 4. Resonant frequency information derived using the Bergh and Tijdeman code for a family of such systems is presented in Table II ( the tube length of the systems is $1 \mathrm{~cm}$, and the helium temperature is $300 \mathrm{~K}$. ) Primary resonance is calculated as a function of volume ratio. Using this information, the helium-specific equation will be formulated empirically.

\begin{tabular}{cc}
$\begin{array}{c}\text { VOLUME } \\
\text { RATIO }\end{array}$ & $\begin{array}{l}f_{0} \\
{[\mathrm{~Hz}]}\end{array}$ \\
\hline .01 & $2.583 E 4$ \\
.1 & $2.258 \mathrm{E} 4$ \\
.5 & $1.537 \mathrm{E} 4$ \\
1.0 & $1.181 \mathrm{E} 4$ \\
2.0 & $8.739 \mathrm{E} 3$ \\
3.0 & $7.247 \mathrm{E} 3$ \\
5.0 & $5.687 \mathrm{E} 3$ \\
7.5 & $4.673 \mathrm{E} 3$ \\
10.0 & $4.058 \mathrm{E} 3$ \\
12.5 & $3.713 \mathrm{E} 3$ \\
15.0 & $3.326 \mathrm{E} 3$
\end{tabular}

TABLE II: BST PREDICTIONS FOR $f_{0}$ IN AN UNDERDAMPED He TRANSMISSION TUBE ( $\left(\mathrm{g}_{\mathrm{g}}^{0}=300 \mathrm{~K}\right.$; $P_{\mathrm{g}}=150 \mathrm{bar} ; \mathrm{L}=1 \mathrm{~cm}$; $D=.325 \mathrm{~cm} ; z=1878$ e $10 \mathrm{OHz}$

Figure 2 is a plot of the computer-predicted resonant frequencies from Table II as a function of volume ratio. Superimposed on the plot is a curve that was empirically derived to fit the points. 


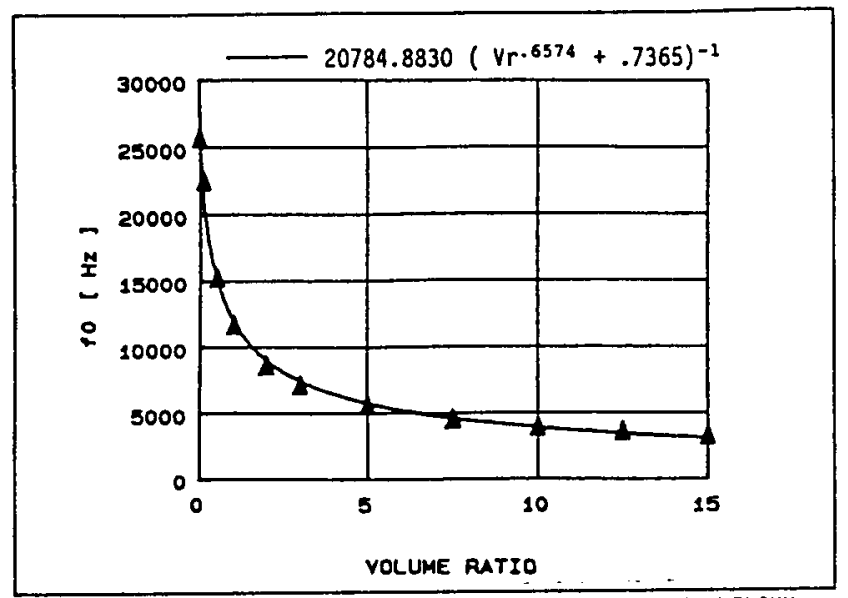
Figure 2: $f_{0}$ vs $v r$ fOR A $1 \mathrm{~cm}$ TUSE WITH UNDERDAMPED HELIUM

An expression for the primary resonant frequency of any underdamped helium transmission tube can now be deduced by forcing the curve-fit equation into the mathematical form of equation 4 :

$$
\begin{aligned}
f_{0}(\mathrm{~Hz}) & \simeq 20784.8830\left(\mathrm{Vr}^{.6574}+.7365\right)^{-1} \\
& =1200 \frac{\sqrt{300 \mathrm{~K}}}{1 \mathrm{~cm}} \frac{1}{\left(\mathrm{Vr}^{.6574}+.7365\right)} \\
& =1200 \frac{\sqrt{\mathrm{T} g_{\mathrm{g}}(\mathrm{K})}}{\mathrm{L}(\mathrm{cm})} \frac{1}{\left(\mathrm{Vr}^{.6574}+.7365\right)}
\end{aligned}
$$

[ eq. 5 ]

Table III lists the resonant frequencies calculated using this equation alongside the B\&T predictions for several underdamped helium systems. Good

\begin{tabular}{|c|c|c|c|c|c|c|c|}
\hline$\left[\begin{array}{c}\mathrm{L} \\
\mathrm{cm}]\end{array}\right]$ & {$\left[\begin{array}{c}0 \\
{[\mathrm{~cm}]}\end{array}\right]$} & $v_{r}$ & {$\left[\begin{array}{c}k_{0} \\
{[}\end{array}\right.$} & {$\left[\begin{array}{c}P . \\
\text { [ bar ] }\end{array}\right.$} & $\left.\begin{array}{c}\mathrm{f}_{1}: \mathrm{Hz} \\
{[\mathrm{B \& T}]}\end{array}\right]$ & $\begin{array}{c}\mathrm{I}_{0}: \mathrm{Hz} \\
{[\mathrm{EO} .5]}\end{array}$ & DEVIATION \\
\hline 1.0 & .16 & .25 & 300 & 100 & 18650 & 18256 & $-2.1 \mathrm{X}$ \\
\hline 1.5 & .16 & 2.5 & 300 & 150 & 5250 & 5406 & $3.0 x$ \\
\hline 2.0 & .32 & 0.5 & 300 & 200 & 7748 & 7583 & $-2.1 x$ \\
\hline 2.5 & .32 & 5.0 & 500 & 150 & 2888 & 2967 & $2.7 x$ \\
\hline 3.0 & .64 & .75 & 750 & 150 & 6830 & 7003 & $2.5 x$ \\
\hline 5.0 & .64 & 7.5 & 1050 & 150 & 1701 & 1729 & $1.6 \%$ \\
\hline 10.0 & .64 & 10.0 & 300 & 150 & 405 & 394 & $-2.7 x$ \\
\hline
\end{tabular}
correlation exists between predictions of equation 5 and the computer results.

TABLE III: COMPARISON OF EQUATION 5 HITH B8T PREDICTIONS FOR 7 TRANSMISSION TUBES HITH UNDERDAMPED HELIUM

Equation 5 is therefore a legitimate predictor of $f_{0}$ in pressure transmission tubes which incorporate underdamped helium. Since it explicitly includes a volume-ratio dependence, it will be more generally valid than the conventional 'quarter wave' equation for acoustic resonance of a closed-ended tube.

\section{PRESSURE AMPLITUDE DISTORTION}

In engineering problems which can be analyzed as second order systems, a knowledge of the first resonance will be sufficient to characterize amplitude response at frequencies that are low with respect to $f_{0}$. In other words, although $f_{0}$ will shift as system parameters are varied, the amplitude distortion at some low frequency, $f$, will remain a fixed function of $f / f_{0}$.

Frequency response information for a family of underdamped helium systems is presented in Table IV. Entries in the table indicate that the tube and volume systems under consideration exhibit this second-order property of amplitude response.

\begin{tabular}{|l|l|l|l|l|l|l|l|}
\hline$V_{r}$ & $\begin{array}{c}f_{0} \\
{[\mathrm{~Hz}]}\end{array}$ & $\begin{array}{c}f 10 \% \\
{[\mathrm{~Hz}]}\end{array}$ & $\frac{f 10 \%}{f_{0}}$ & $\begin{array}{c}f 5 \% \\
{[\mathrm{~Hz}]}\end{array}$ & $\frac{f 5 \%}{f_{0}}$ & $\begin{array}{c}f 1 \% \\
{[\mathrm{~Hz}]}\end{array}$ & $\frac{f 1 \%}{f_{0}}$ \\
\hline .01 & $2.583 E 4$ & $7.056 E 3$ & .273 & $5.085 E 3$ & .197 & $2.308 E 3$ & .089 \\
\hline .1 & $2.258 E 4$ & $6.239 E 3$ & .276 & $4.493 E 3$ & .199 & $2.040 E 3$ & .090 \\
\hline .5 & $1.537 E 4$ & $4.396 E 3$ & .286 & $3.169 E 3$ & .206 & $1.440 E 3$ & .094 \\
\hline 1.0 & $1.181 E 4$ & $3.450 E 3$ & .292 & $2.485 E 3$ & .210 & $1.133 E 3$ & .096 \\
\hline 2.0 & $8.739 E 3$ & $2.588 E 3$ & .296 & $1.870 E 3$ & .214 & $8.484 E 2$ & .097 \\
\hline 3.0 & $7.247 E 3$ & $2.157 E 3$ & .298 & $1.559 E 3$ & .215 & $7.103 E 2$ & .098 \\
\hline 5.0 & $5.687 E 3$ & $1.701 E 3$ & .299 & $1.231 E 3$ & .216 & $5.586 E 2$ & .098 \\
\hline 7.5 & $4.673 E 3$ & $1.404 E 3$ & .300 & $1.012 E 3$ & .217 & $4.599 E 2$ & .098 \\
\hline 10.0 & $4.058 E 3$ & $1.221 E 3$ & .301 & $8.802 E 2$ & .217 & $4.000 E 2$ & .099 \\
\hline 12.5 & $3.713 E 3$ & $1.118 E 3$ & .301 & $8.046 E 2$ & .217 & $3.673 E 2$ & .099 \\
\hline 15.0 & $3.326 E 3$ & $9.977 E 2$ & .300 & $7.231 E 2$ & .217 & $3.287 E 2$ & .099 \\
\hline
\end{tabular}

TABLE IV: BST FREQUENCY RESPONSE PREDICTIONS FOR A PRESSURE TRANSMISSION TUBE WITH UNDERDAMPED HELIUM ( $T=$ $300 \mathrm{~K} ; P_{g}=150 \mathrm{bar} ; \quad L=1 \mathrm{~cm} ; \quad D=.325 \mathrm{~cm} ; z=18780100 \mathrm{H}$ )

The columns labelled $f_{103}, f_{5,}$ and $f_{13}$ in the table represent the frequencies at which the amplitude ratio becomes $1.10,1.05$ and 1.01 respectively. Independent of the volume ratio, these frequencies are essentially fixed percentages of the resonant frequency. Specifically:

$$
\begin{aligned}
& f_{1 v} \simeq .10 \mathrm{f}_{0} \\
& \mathrm{f}_{31} \simeq .21 \mathrm{f}_{0} \\
& \left.\mathrm{f}_{101} \simeq . \mathrm{eq.}\right]
\end{aligned}
$$

Dynamic pressure signals oscillating at frequencies below $f_{1}$ will pass the transmission line with an amplitude distortion of less than 16. Similar definitions hold for $f_{5}$ and $f_{10}$. Moreover, linear interpolation can be used to approximate intermediate values of amplitude distortion. For example, $f_{34}$ can be interpolated between $f_{31}$ and $f_{54}$ :

$$
f_{31} \simeq f_{11}+\frac{1}{2}\left(f_{31}-f_{18}\right) \simeq .15 f_{0}[\text { eq. } 9]
$$

Thus, after calculating the resonant frequency for a given pressure measurement system, error bands on the amplitude distortion are easily established.

Consider a hypotherical problem from the instrumentation designer's point of view: It is necessary to measure the amplitude of dynamic 
pressures in a $100 \mathrm{~Hz}$ engine to within 3z; Then, $f_{32}$ is taken to be $100 \mathrm{~Hz}$. Based on the result of equation 9 , the transmission lines must be designed to resonate at $f_{0} \geq f_{34} \div .15=667 \mathrm{~Hz}$. The higher $f_{0}$ can be made, the better in terms of pressure amplitude integrity.

\section{PRESSURE PHASE DISTORTION}

In second order systems that are completely undamped, there will be no phase shift at frequencies below the primary resonance. In underdamped systems, the phase shifts can generally be made small by careful system design. This result is particularly significant to FPSE research, where it is often necessary to resolve the pressure phases to within one tenth of one degree.

Unfortunately, the detailed analysis of phase distortion is somewhat more complicated than that of amplitude distortion. The phase shift will depend not only the factor $f / f_{0}$, but also on the amount of damping present in the transmission system. (In the case of amplitude distortion, as long as the system is underdamped, the actual amount of damping is unimportant to low-frequency response.)

The appendix offers a heuristic development of phase distortion behavior in underdamped systems. The resulting approximation describes the functionality of $\phi$ for a fixed volume-ratio:

$$
\phi \quad a \quad \frac{L^{2} f^{1.5}}{D \sqrt{P_{g}}} \quad[\text { eq. } 10]
$$

Table $V$ presents Bergh and Tijdeman predictions for the phase distortion in a specific underdamped helium transmission tube. ( $L$ is $1 \mathrm{~cm}, D$ is $.325 \mathrm{~cm}$ and $P_{\text {g }}$ is 150 bar.) The phase shifts for a pressure oscillating at $100 \mathrm{~Hz}$ are tabulated as a function of volume ratio.

\begin{tabular}{cl}
\hline VOLUME RATIO & $\phi$ e $100 \mathrm{~Hz}$ \\
\hline .01 & $-.00006^{\circ}$ \\
0.1 & $-.00007^{\circ}$ \\
0.5 & $-.00012^{\circ}$ \\
1.0 & $-.00019^{\circ}$ \\
2.0 & $-.00027^{\circ}$ \\
3.0 & $-.00042^{\circ}$ \\
5.0 & $-.00064^{\circ}$ \\
7.5 & $-.00093^{\circ}$ \\
10.0 & $-.00121^{\circ}$ \\
12.5 & $-.00144^{\circ}$ \\
15.0 & $-.00179^{\circ}$
\end{tabular}

TABLE V: B\&T PREDICTIONS FOR $\phi$ Q100Hz IN AN UNDERDAMPED HELIUM TRANSMISSION TUBE ( $P_{g}=150 \mathrm{bar}: T_{g}=300 \mathrm{~K}$;
Using the scaling rules of equation 10 , information In Table $v$ can be applied generally to any underdamped helium system as follows:

$$
\begin{aligned}
\phi_{\text {ganeral }} & \quad \frac{(\mathrm{L} / 1 \mathrm{~cm})^{2}(\mathrm{f} / 100 \mathrm{~Hz})^{1.5}}{(\mathrm{D} / .325 \mathrm{~cm}) \sqrt{\mathrm{P}_{\mathrm{g}} / 150 \mathrm{bar}}} \phi_{\text {table }} \\
& =3.98 \mathrm{E}-3 \frac{\mathrm{L}^{2} \frac{\mathrm{f}^{1.5}}{\mathrm{D} \sqrt{\mathrm{P}_{\mathrm{g}}}} \phi_{\text {table }}}{\text { [eq. } 11]}
\end{aligned}
$$

Although the absolute values of $\phi$ in Table $V$ are orders of magnitude less than $.1^{\circ}$, it can be seen that an imprudent system design will result in unacceptably large phase shifts. Consider, for example, a 150 bar engine operating at $100 \mathrm{~Hz}$. A transmission tube with $L=10 \mathrm{~cm}, D=.16 \mathrm{~cm}$ and $V r=10$ can distort the phase of the pressure signal by as much as $.25^{\circ}$; this by virtue of equation 11 and Table $v$.

The key point here is that phase shifts in underdamped systems are generally small; however, increasing the tube length of the system will magnify the phase distortion by a factor of $\mathrm{L}^{2}$.

\section{LIMITS OF APPLICABILITY}

The specific guidelines derived in this paper apply exclusively to volume-terminated transmission tubes which incorporate underdamped helium as the internal fluid. There are, moreover, underlying assumptions built into Iberall's fundamental mathematics which further limit the applicability of the results.

As one notable example, the fundamental flow equations at the root of the analysis have been linearized based on an assumption that dynamic pressure amplitudes are small compared to the mean pressure. Fortunately, Iberall addresses the implications of this assumption for situations where the pressure amplitude is large with respect to mean pressure. Specifically, the fundamental of a large pressure oscillation will be transmitted in accordance with the linearized-model predictions; however, higher-order harmonic distortion may be artificially induced in the pressure measurement. Therefore, in FPSE research, where dynamic pressure amplitudes as large as 207 of the mean pressure are not uncommon, harmonic distortion in measured pressure signals may be a phantom of the transmission line.

Another shortcoming of the theory is its inability to account for complicated thermal interactions between the gas and the tube. If, for example, dynamic pressure in a FPSE's high-temperature expansion space is being measured by a water-cooled transducer at the end of a transmission tube, then severe temperature gradients will be induced along the tube. The extent of resulting anomalies in the pressure measurement cannot be predicted using methods based on Iberall's classical analysis. 


\section{CONCLUDING REMARKS}

At the NASA Lewis Research Center, there is an effort in progress to examine a more generalized solution to the tube and volume response problem using state of the art computational fluid dynamics methods. In conjunction, an instrumentation research program is under development to provide experimental verification of the improved analysis.

Until results from this effort are available, the classical analysis should be applied with conservatism. In practice, the Iberall theory can provide estimated error bands for amplitude distortion and phase shift; however, it is not adequate for calculating reliable correction factors to compensate for errors in the pressure measurement.

There is one conclusion that has been implicit throughout this paper: Flush-mounting a pressure transducer, whenever possible, is preferred. This circumvents transmission line distortion, and it adds no unwanted dead-volume into the FPSE's working space.

When a flush-mount is not possible, then careful design of a pressure transmission system is imperative. This paper has developed some basic guidelines for instrumentation engineers to follow in the early design stages of a pressure measurement system. To summarize in general: the tube diameter should be large enough to ensure underdamped helium flow; and both volume-ratio and tube length should be minimized in order to maximize $f_{0}$ with respect to $f_{\text {ong }}$.

The guidelines are approximate, and should be viewed as a starting point; they are no substitute for a full dynamic-response analysis. The computer codes cited in this paper are highly recommended; If properly used, they should prove invaluable to the instrumentation designer. Hopefully, the basic properties of dynamic response described in this paper will facilitate an efficient use of the analytical codes, and will aid in the effective design of pressure measurement systems. In free piston stirling engine research, the importance of maintaining rigorous standards in the development of instrumentation systems cannot be overstated.

\section{APPENDIX: PHASE DISTORTION FOR UNDERDAMPED FLOW}

Two factors contribute to phase distortion in an underdamped transmission system at low frequencies with respect to $f_{0}$. This appendix will examine these factors for systems with fixed volume-ratios.

One factor will vary as a function of $f / f_{0}$; an effect characteristic to both phase and amplitude distortion. For phase, the function is nearly linear in the low frequency region. In this appendix, the first factor will be denoted by $\lambda$. Using equation 4 with a fixed volume-ratio:

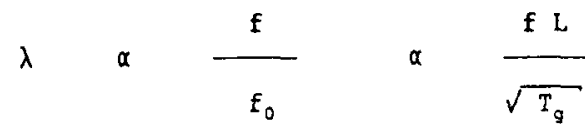

The second factor represents the effect of damping on the phase shift. This factor will vary as the square root of the Iberall attenuation parameter $\chi(1):$

$\left.\begin{array}{lll}\sqrt{\chi} & \alpha \quad \sqrt{\mu \mathrm{f} / \mathrm{P}_{\mathrm{g}}} & (\mathrm{L} / \mathrm{D}\end{array}\right)$

( Through mathematical manipulation, the parameter $\chi$ can be expressed in terms of $1 / z$; thus, as expected, high values of $z$ express conditions of low damping. This is left as an exercise for the interested reader. )

Next, $\beta$ will be replaced by the approximation $\beta \cdot T_{g}{ }^{n}$ ( where $\beta$ and $n$ are constants, and $n$ is close to. but less than 1.) Then, combining the two phasedistortion factors leads to:

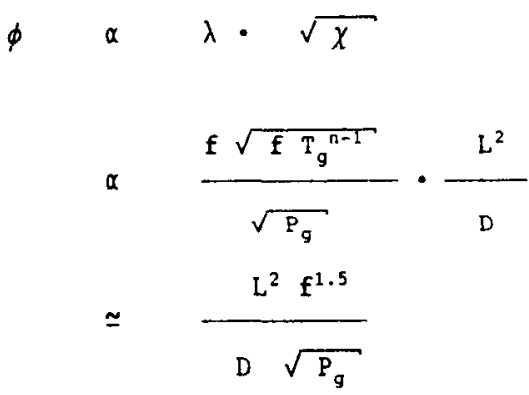

Although this result is approximate, it provides an extremely useful description of the phase sensitivity to transmission tube parameters.

\section{REFERENCES}

[1] Iberal1, Arthur S.: 'Attenuation of Oscillatory Pressures in Instrument Lines', Journal of Research of the National Bureau of Standards, vol. 45, no. 1, July 1950, pp. 85-108.

[2] Goldschmied, F. R.: 'On the Dynamic Response of Viscous Compressible Fluids in Rigid Tubes with Volume Termination as a Function of the Stokes Number', NASA TM X-53785, Sept. 1968.

[3] Nyland, T. W.; Englund, D. R.; Anderson, R. C.: - On the Dynamics of Short Pressure Probes: Some Design Factors Affecting Frequency Response'. NASA TN D-6151, Feb. 1971.

[4] Bergh, H.; Tijdeman, H.: Theoretical and Experimental Results for the Dynamic Response of Pressure Measurement Systems', rep. NLR-TRF.238, National Aero- and Astronautical Research Institute, Amsterdam, Jan. 1965. 


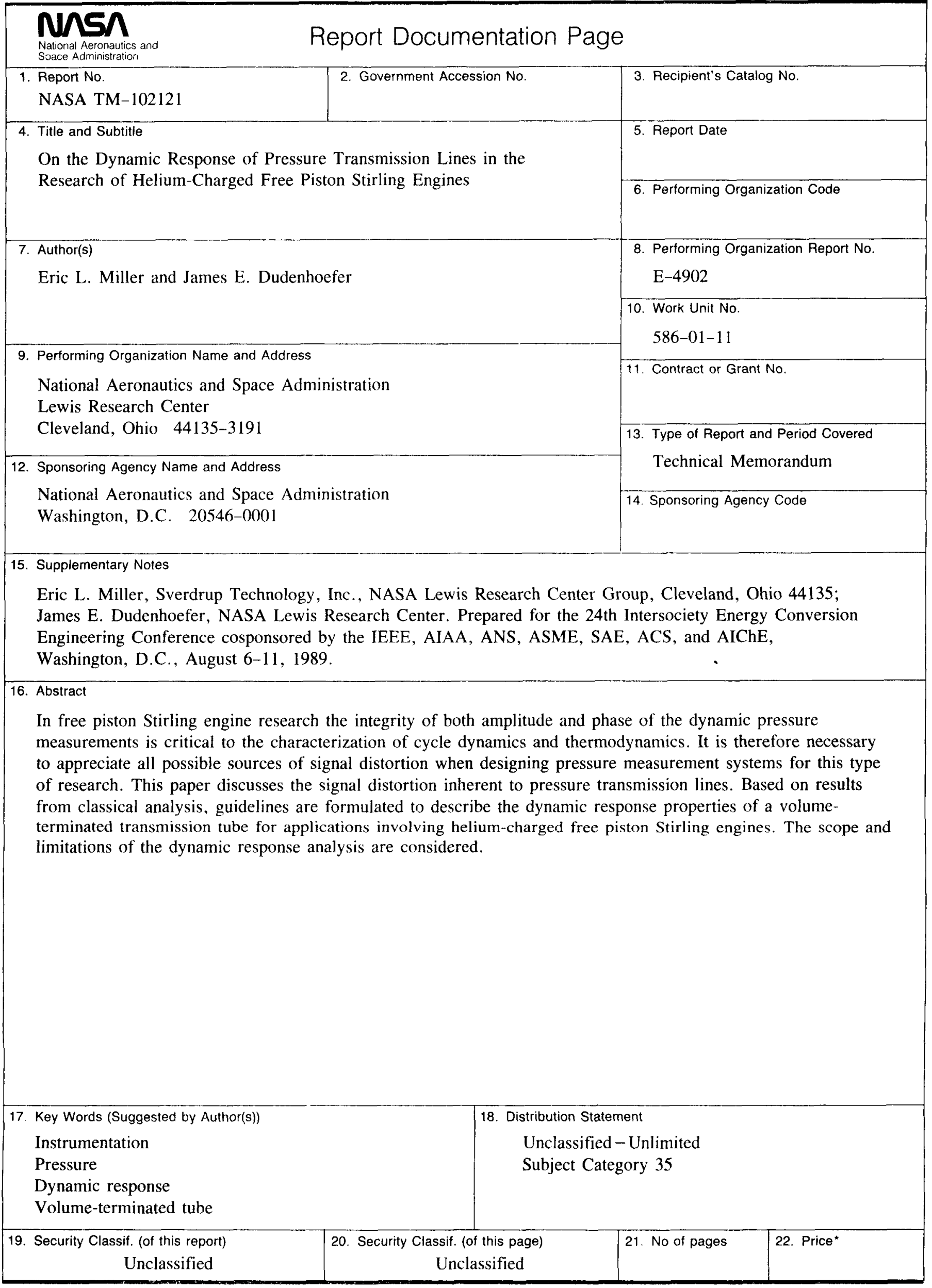

